\title{
LA CREACIÓN Y LA EVOLUCIÓN DE UNASUR
}

J oão Carlos Amoroso Botelho

\begin{abstract}
Resumo
A pesar de que las tentativas de integración en América del Sur remonten al siglo XIX, apenas en 2004, fue creado el primer bloque para reunir los 12 países de la región, la CASA, que pasó a llamarse UNASUR en el 2007. Por qué esta conformación ocurrió en el 2004? La hipótesis es que la convergencia de posiciones integracionistas en las tres potencias regionales, sobre todo en Brasil y Venezuela, impulsada por un canciller y dos presidentes, posibilitó la creación del organismo. Para sostener esto, el trabajo se apoya en una serie de datos empíricos desde los regimenes militares en Argentina y Brasil.
\end{abstract}

Palavras-chave: Integración Regional; UNASUR; Argentina; Brasil; Venezuela.

\begin{abstract}
Despite attempts to integration in South America date back to the nineteenth century, only in 2004, was created the first block to gather the 12 countries of the region, the CASA, which changed the name to UNASUR in 2007. Why this conformation occurred in 2004? The hypothesis is that the confluence of integrationist positions in the three regional powers, especially in Brazil and Venezuela, driven by one chancellor and two presidents, enabled the creation of the body. To sustain this, the work uses a series of empirical data since the military regimes in Argentina and Brazil.
\end{abstract}

Key-words: Regional Integration; UNASUR; Brazil; Argentina; Venezuela.

\section{Introducción}

Las tentativas de cooperación e integración en América del Sur remontan al siglo XIX. Sin embargo, apenas en el 2004, fue conformado el primer bloque para reunir los 12 países de la región, la CASA (Comunidad Sudamericana de Naciones), que pasó a llamarse UNASUR (Unión de Naciones Sudamericanas) en el 2007. ¿Por qué el organismo surgió en el 2004?

La CASA fue creada por la Declaración de Cusco, de 8 de diciembre de 2004, en la III Cumbre Sudamericana de Presidentes, en la ciudad peruana. El 23 de mayo de 2008, fue firmado en Brasilia, Brasil, el Tratado Constitutivo de la UNASUR, que le confiere carácter jurídico internacional. El objetivo del bloque, que a partir de ahora pasará a ser aquí apenas UNASUR, es 
construir, de maneira participativa e consensuada, um espaço de integração e união, no âmbito cultural, social, econômico e político entre seus povos, priorizando o diálogo político, as políticas sociais, a educação, a energia, a infra-estrutura, o financiamento e o meio ambiente, entre outros, com vistas a eliminar a desigualdade socioeconômica, alcançar a inclusão social e a participação cidadã, fortalecer a democracia e reduzir as assimetrias no marco do fortalecimento da soberania e independência dos Estados (Tratado Constitutivo da União de Nações Sul-Americanas, $2008^{1}$ ).

La historia de América Latina, sobre todo a partir de la década de 1960, está tomada de ejemplos de tentativas de cooperación e integración, de ámbito regional o subregional. Con la entrada de México a principios de los años 1990 en el NAFTA (North America Free Trade Agreement), la cancillería brasileña, que ya priorizaba iniciativas con participación de menos países, ha tenido una justificativa para concentrarse en América del Sur, como afirma el ex secretario general del Itamaraty Luiz Felipe de Seixas Corrêa. "O MERCOSUL, o mais bem concebido de todos os mecanismos de integração, alavancou a idéia de América do Sul, região de convergência política e de integração econômica, em substituição à América Latina, desde que o México se orientou para o norte" (CORRÊA, 2007, XIII).

El período priorizado empieza con la serie de reconquistas de la democracia y el fin del último ciclo de regímenes militares en América del Sur y sigue hasta la actualidad. Los países en que el estudio se concentrará son Argentina, Brasil y Venezuela, consideradas aquí las potencias regionales. Era imperativo, ya que son 12 naciones, priorizar algunas. Así, hubo la elección con base en aspectos de orden político, económico y geográfico. Argentina y Brasil se han tornado, por sus dimensiones territoriales y poderíos económico y político desarrollados, los protagonistas en el subcontinente. Había, sin embargo, la necesidad de incluir un representante del norte de la región. El elegido fue Venezuela, en detrimento de Colombia, por tres razones: 1) ejerce más

\footnotetext{
$1 \quad$ Disponible en: <http://www.mre.gov.br/ portugues/imprensa/nota_detalhe3.asp?ID_RELEASE=5466>. Acceso en: 24 may. 2008.
}

REVISTA DEBATES, Porto Alegre, v. 2, n. 2, p. 299-324, jul.-dez. 2008.300 
protagonismo político, por el peso que ha obtenido su presidente desde 1999; 2) con el alza del petróleo, ha pasado a hacer préstamos e inversiones en otros países sudamericanos; y 3) su economía es más grande que la colombiana.

La hipótesis es que la convergencia de posiciones integracionistas en las tres potencias, impulsada por un canciller y dos presidentes, posibilitó la formación del bloque. El factor más importante ha sido la acción gubernamental, sobre todo de Brasil y Venezuela, para superar obstáculos persistentes y promover la integración, aprovechando una coyuntura favorable.

\section{Marco Teórico}

Haas (1958) define integración como el proceso en que los actores políticos en diversos países son persuadidos a transferir sus lealtades, expectativas e actividades a un nuevo centro, cuyas instituciones procesan o demandan jurisdicción sobre los Estados. Este concepto es útil porque engloba un proceso social e otro político, pero no se seguirá la línea que esta definición reflecte. Para la situación sudamericana, es necesario limitarse a las percepciones e actitudes hacia la integración y a las políticas conducidas en la dirección de la construcción y la consolidación de instituciones supranacionales.

El neofuncionalismo, del cual viene la definición citada arriba, identifica dos componentes para la integración. El punto de partida se da con la demanda de la sociedad. Esto, sin embargo, no es suficiente, porque la demanda no se convierte automáticamente en resultados. EI segundo aspecto consiste en el poder de los especialistas y de las organizaciones supranacionales para estructurar la agenda y intermediar acuerdos. Esta vertiente presenta problemas para explicar cuales grupos son exitosos en afectar las políticas y tiene una concepción tecnocrática de la resolución de las cuestiones en el ámbito supranacional (CAPORASO, 1998). Además, reduce el papel de los Estados.

Ya para el intergubernamentalismo, la teoría de la integración que más se utiliza del neorrealismo, los atores preponderantes en el sistema internacional son los Estados, que actúan racionalmente según 
sus intereses y capacidad de acción. Ellos pueden incorporarse a iniciativas de cooperación e aceptar la creación de organizaciones supranacionales, pero con determinados objetivos y sin perder el control sobre ellas. De acuerdo con lo que plantea esta corriente, un proceso de aproximación solo puede ser intergubernamental y nunca trascenderá la autoridad de los Estados (SCHMITTER, 1996).

El intergubernamentalismo liberal es una variante que sitúa la integración en tres dimensiones, la formación de preferencias en la sociedad, la negociación entre los gobiernos y la construcción de instituciones supranacionales. Así, gobiernos que son actores racionales y perseguidores de intereses económicos capturan las demandas de la sociedad, definen un programa, negocian diferencias con otros países de acuerdo con la importancia que confieren al tema y su capacidad de imponerse y se comprometen con reglas e organizaciones supranacionales para evitar que los acuerdos no sean cumplidos. El problema de esta corriente es que ve las instituciones supranacionales como agentes pasivos y limitados a reducir costes de transacción y aumentar la eficiencia de los acuerdos (CAPORASO, 1998). Además, deja los intereses políticos de los Estados en segundo plano.

El abordaje será intermedio entre neofuncionalismo e intergubernamentalismo, las principales teorías que explican la creación y los primeros pasos de un proceso de integración. Con esto, se evitará al mismo tiempo minimizar y maximizar el papel de las instituciones supranacionales. No se puede ignorar su actuación, pues siempre está presente, una vez que el simple hecho de que un Estado acepte tomar parte de un proceso de integración ya implica en una cesión de soberanía a favor de alguna forma de institución supranacional, sea regla, organización o los dos. Tampoco se puede sobrevalorar su importancia, sobre todo para procesos poco desarrollados como el sudamericano.

Así, para el tipo de evaluación de UNASUR que se hará, no es posible se apoyar únicamente en el neofuncionalismo, pues el estadio del proceso no condice con un énfasis en las instituciones supranacionales y con la definición de un papel secundario a los Estados, 
aspectos característicos de la teoría neofuncionalista. Al mismo tiempo, esta corriente tiene que ser llevada en cuenta, porque el entramado institucional de UNASUR está en evolución, reflectando la actuación de los Estados miembros para empezar a conferir autonomía al proceso, y precisa ser considerado y analizado.

El intergubernamentalismo dará el marco de análisis, ya que el papel de los Estados es lo más importante en el período enfocado. Sin embargo, tampoco podrá ser la única teoría considerada, pues presenta problemas para tratar de procesos de integración que empiezan a institucionalizarse y a tener transferencia de soberanía, ya que no admite que reglas $u$ organizaciones supranacionales transciendan la autoridad de los Estados.

El intergubernamentalismo liberal, que en sus tres dimensiones de análisis de la integración combina bajo una base racionalista elementos de las teorías liberal, realista y funcionalista de las relaciones internacionales, se presenta como una solución conciliatoria entre el intergubernamentalismo e el neofuncionalismo, pues valoriza el papel de los Estados e al mismo tiempo admite la transferencia de soberanía. Sin embargo, tampoco podrá ser utilizado integralmente, porque además de dar poca importancia a las instituciones supranacionales y los intereses políticos de los Estados, tiene otro problema para su aplicación al caso sudamericano: las demandas de las sociedades nacionales no han sido decisivas en la actuación de los gobiernos, a causa de la escasa participación social hasta ahora en el proceso, sea de grupos organizados o no.

La historia de las relaciones internacionales es otro campo de estudios en que se buscará apoyo. Cervo (2007) identifica seis obstáculos que han dificultado las tentativas de integración en América Latina: las rivalidades; las ambiciones nacionales excesivas; las posturas frente a Estados Unidos; las percepciones sobre Brasil en América hispánica; la desatención de los gobiernos a las posiciones en las sociedades; y la diplomacia de la obstrucción. 


\section{La Creación de UNASUR}

Efectivamente, UNASUR fue creada en el 2004, pero el inicio del proceso que ha contribuido a esto puede ser situado en el fin de los regímenes militares en Argentina y Brasil, a mediados de la década de 1980, y incluso un poco antes, a finales de los años 1970, con las dictaduras todavía en vigor.

El Acuerdo Tripartito, entre Argentina, Brasil y Paraguay en el 1979, fue un paso en la aproximación entre los dos primeros países que, superando divergencias persistentes sobre el aprovechamiento de los ríos de la Bacía del Plata, contribuyó no apenas para la ulterior creación de UNASUR como antes en la del MERCOSUR (Mercado Común del Sur). En el año siguiente, el presidente de Brasil, el general João Baptista Figueiredo, realizó una visita a Buenos Aires, la primera de un jefe de Estado brasileño desde 1935, y firmó una serie de protocolos de cooperación, incluso en las áreas militar y de energía nuclear. Con la desindustrialización promovida por la dictadura argentina, Brasil se consolidó entre 1980 y 1984 como el segundo abastecedor del vecino.

En el 1985, con los dos países ya redemocratizados, los presidentes Raúl Alfonsín y José Sarney firmaron la Declaración de Iguazú. Después, el Tratado de Integración, Cooperación y Desarrollo, de 1988, previó la creación de un espacio económico común en el plazo de 10 años, lo que fue adelantado para 1994 por la Acta de Buenos Aires, firmada en el 1990 por Carlos Menem y Fernando Collor. Uruguay y Paraguay pronto se juntaron, resultando en la creación del MERCOSUR por el Tratado de Asunción, en el 1991. Con Venezuela, Brasil firmó el Compromiso de Caracas durante el gobierno Sarney.

Sin embargo, la diseminación de reformas neoliberales en la región entre finales de los años 1980 y principios de la década siguiente desencadenó en las políticas externas la estrategia de agradar a los norteamericanos para buscar ventajas. También implicó en la valorización del papel del mercado y de la globalización. Así, el énfasis transitó del nacional y del regional para el global. En la Argentina de Menem, la prioridad fue para el establecimiento de relaciones especiales 
con Estados Unidos. Al mismo tiempo, el presidente argentino enfatizaba la integración en el Cono Sur, como forma de crear una contrapeso y aumentar su poder de negociación con los norteamericanos. En Brasil, Collor también buscaba su versión de las relaciones especiales con Estados Unidos, pero no sus sucesores en los años 90. En Venezuela, las gestiones de Carlos Andrés Pérez y Rafael Caldera adoptaran la política de apertura e internacionalización del sector petrolífero, lo que coincidía con los intereses norteamericanos.

La aparente contradicción de que el MERCOSUR haya sido creado por dos presidentes en Argentina y Brasil que adoptaron reformas neoliberales y que buscaban aproximarse de los Estados Unidos se resuelve con una mirada más atenta. La creación de un bloque con la énfasis en el aspecto comercial e en la apertura de mercados se encajaba perfectamente con la línea económica que los gobiernos argentino y brasileño empezaban a adoptar y les confería credibilidad para seguir con las reformas (COSTA VAZ, 2002).

En Brasil, las circunstancias ya cambiaron con la caída de Collor y la ascensión de Itamar Franco en el 1992. En su gobierno, el país propuso oficialmente por primera vez, en el 1993, la creación de un organismo con todas las naciones sudamericanas, la ALCSA (Área de Libre Comercio Sudamericana). Todavía se trataba de un bloque comercial, pero ya era un nuevo movimiento en el sentido de promover y ampliar la unión regional. Incluso, el canciller brasileño de entonces era Celso Amorim, lo mismo que volvería al cargo en el 2003, con la llegada de Luiz Inácio Lula da Silva al poder, y capitanearía la creación de UNASUR.

En Argentina, el fin de la gestión Menem en el 1999 no mejoró la situación para la integración, porque Fernando de la Rúa tuvo un mandato efímero y turbulento. Así, la confluencia de posiciones integracionistas en detrimento de las relaciones especiales con Estados Unidos, que había en el Brasil de Fernando Henrique y en la Venezuela de Chávez desde el mismo año de 1999, no pudo contar con el refuerzo argentino. A partir de 2002, cuando Eduardo Duhalde llegó al poder, el escenario se volvió más favorable para la unión regional, pues con él, 
Fernando Henrique y Chávez, las tres potencias pasaran a tener gobiernos inclinados a la integración. Este cuadro se reforzó en el 2003 con las tomas de posesión de Lula en Brasil y Néstor Kirchner en Argentina.

Por otro lado, persisten obstáculos históricos que han dificultado la integración. De los seis que Cervo (2007) apunta, al menos cinco todavía se manifiestan en mayor o menor grado: las rivalidades; las ambiciones nacionales excesivas; las posturas frente a Estados Unidos; las percepciones sobre Brasil en América hispánica; y la diplomacia de la obstrucción. Además, parecer haber en la política exterior norteamericana la intención de crear divisiones y dificultar la formación de bloques en América del Sur. Una evidencia de esto es que, con el fracaso de las negociaciones de la ALCA (Área de Libre Comercio de las Américas), el gobierno Bush ha empezado a ofrecer acuerdos bilaterales de libre comercio a países sudamericanos.

Mismo con las dificultades citadas, el cuadro en las tres potencias regionales se ha mostrado más favorable a la integración sudamericana a partir de 2002, con la toma de posesión de Duhalde y la continuidad de los gobiernos Fernando Henrique y Chávez. A los dos primeros, pronto se siguieron Kirchner y Lula, generando perspectivas todavía más prometedoras al proceso. Desde entonces, han ocurrido la creación de UNASUR y la adhesión en el 2006 de Venezuela al MERCOSUR como miembro efectivo, lo que todavía requería la aprobación de los Parlamentos de los cuatro integrantes.

Fue a partir del proceso de reuniones presidenciales, iniciado en el 2000, que se llegó a la creación de UNASUR, en la III Cumbre Sudamericana de Presidentes, en el 2004. Lula y su canciller capitanearon la iniciativa, a partir de sugerencia del entonces presidente peruano, Alejandro Toledo. Desde 2004, han ocurrido tres Cumbres de Jefes de Estado, en el 2005 en Brasilia, en el 2006 en Cochabamba, Bolivia, e en el 2008 nuevamente en Brasilia, cuando se firmó el Tratado Constitutivo. En el 2007, se cambió el nombre de la CASA para UNASUR. 


\section{Las Variables}

La creación de UNASUR pasará ahora a ser explicada a partir de tres variables independientes: 1 ) las políticas externas de las tres potencias regionales para la integración; 2) la evolución de las relaciones entre Argentina y Brasil; 3) y la ascensión de un líder en Venezuela con intención y condiciones financieras para impulsar el proceso.

Las tres variables independientes están asociadas a la acción gubernamental. Esto se explica por el período enfocado y las características del caso. Como la intención es explicar el surgimiento del organismo, no se puede considerar el papel de un entramado institucional que todavía no existía. Tampoco es representativa la agregación de preferencias sobre el proceso en las sociedades nacionales. Mismo después de creada UNASUR, ha sido escasa hasta ahora la participación de grupos de la sociedad civil, organizados o no.

Hay un aspecto ya citado que también tiene influencia en los planos de integración en América del Sur: la actuación de Estados Unidos para dificultar el proceso. Este punto tiene que ser llevado en cuenta, pero no merece la condición de variable independiente, pues los factores endógenos parecen ser más importantes.

Los próximos pasos serán justificar cada variable y relacionar los indicadores que sustentan la hipótesis de que la convergencia de posiciones integracionistas en las tres potencias regionales, impulsada por un canciller y dos presidentes, posibilitó la creación de UNASUR.

\section{Las Políticas de las Tres Potencias para la I ntegración}

Por medio de la política externa, los Estados actúan en el escenario internacional. Como se considera aquí que la acción gubernamental es determinante para explicar el objeto de estudio, las políticas externas para la integración regional tienen que ser una variable independiente. $Y$ una vez que se cree que Argentina, Brasil y Venezuela dictan el rumbo del proceso sudamericano, hay que se evaluar las políticas externas de los tres para la integración. 
En Brasil, las circunstancias se han mantenido más favorables a la integración desde la caída de Collor en el 1992. Lo mismo se pasó con Venezuela a partir de la ascensión de Chávez en el 1999, a pesar de sus problemas internos, sobre todo en el 2002, con un golpe de Estado y el inicio de una paralización general, e en el 2004, con un referendo sobre su mandato. La convergencia de posiciones integracionistas en las tres potencias regionales tardó a completarse hasta la llegada de Duhalde al poder en el 2002. Él heredó una situación económica y social crítica y tuvo que concentrarse en la política interna, pero su gobierno no dejó de marcar un cambio de paradigma, después de los años de reformas neoliberales y de replanteamiento en la política exterior de la idea de convertir Argentina en el aliado preferencial de los Estados Unidos en América del Sur.

El panorama para la integración sudamericana se ha tornado más favorable a partir de 2003, con las ascensiones de Kirchner y Lula. Ha sido así sobre todo con Lula, que nombró para canciller el responsable por la propuesta de la ALCSA. Ya Kirchner tuvo que concentrarse en la situación interna hasta lograr en el 2005 la renegociación de la deuda externa en moratoria, pero consolidó el cambio de paradigma de política exterior empezado con Duhalde.

Hay datos para sostener los puntos planteados, aunque se tenga claro que son indicadores y por sí no explican la integración. En el comercio entre Argentina y Brasil, por ejemplo, ha ocurrido aumento de las exportaciones brasileñas en 15 de los últimos 22 años, mientras para las importaciones, en 17 de los 22 (ver Cuadros 1 y 2). Los años de 2001 y 2002 registraron caídas, cuando se pasó el peor de la crisis argentina, pero desde 2003 ha ocurrido una recuperación, con crecimiento de las exportaciones en todos los años y de las importaciones en cuatro. Desde que Lula llegó a la Presidencia en Brasil, el volumen del comercio bilateral pasó de US\$7,09 billones en el 2002 para US\$24,83 billones en el 2007. 
Cuadro 1 - Exportaciones brasileñas a Argentina (variación en \%)

\begin{tabular}{|c|c|c|c|c|c|}
\hline $\mathbf{1 9 8 6}$ & $\mathbf{1 9 8 7}$ & $\mathbf{1 9 8 8}$ & $\mathbf{1 9 8 9}$ & $\mathbf{1 9 9 0}$ & $\mathbf{1 9 9 1}$ \\
\hline 23,73 & 22,62 & 17,75 & $-26,27$ & $-10,66$ & 128,81 \\
\hline $\mathbf{1 9 9 2}$ & $\mathbf{1 9 9 3}$ & $\mathbf{1 9 9 4}$ & $\mathbf{1 9 9 5}$ & $\mathbf{1 9 9 6}$ & $\mathbf{1 9 9 7}$ \\
\hline 105,94 & 20,36 & 13,04 & $-2,29$ & 27,94 & 30,94 \\
\hline $\mathbf{1 9 9 8}$ & $\mathbf{1 9 9 9}$ & $\mathbf{2 0 0 0}$ & $\mathbf{2 0 0 1}$ & $\mathbf{2 0 0 2}$ & $\mathbf{2 0 0 3}$ \\
\hline$-0,31$ & $-20,51$ & 16,28 & $-19,68$ & $-53,16$ & 94,75 \\
\hline $\mathbf{2 0 0 4}$ & $\mathbf{2 0 0 5}$ & $\mathbf{2 0 0 6}$ & $\mathbf{2 0 0 7}$ & \multicolumn{3}{|r}{} \\
\hline 61,74 & 34,36 & 18,22 & 22,81 & \multicolumn{1}{|l}{} \\
\cline { 1 - 3 } & & & &
\end{tabular}

Fuente: Ministerio de Desarrollo, Industria y Comercio Exterior de Brasil.

Cuadro 2 - Importaciones brasileñas de Argentina (variación en \%)

\begin{tabular}{|c|c|c|c|c|c|}
\hline $\mathbf{1 9 8 6}$ & $\mathbf{1 9 8 7}$ & $\mathbf{1 9 8 8}$ & $\mathbf{1 9 8 9}$ & $\mathbf{1 9 9 0}$ & $\mathbf{1 9 9 1}$ \\
\hline 57,19 & $-22,02$ & 23,04 & 75,18 & 13,00 & 14,97 \\
\hline $\mathbf{1 9 9 2}$ & $\mathbf{1 9 9 3}$ & $\mathbf{1 9 9 4}$ & $\mathbf{1 9 9 5}$ & $\mathbf{1 9 9 6}$ & $\mathbf{1 9 9 7}$ \\
\hline 7,60 & 56,92 & 34,77 & 52,69 & 21,71 & 16,69 \\
\hline $\mathbf{1 9 9 8}$ & $\mathbf{1 9 9 9}$ & $\mathbf{2 0 0 0}$ & $\mathbf{2 0 0 1}$ & $\mathbf{2 0 0 2}$ & $\mathbf{2 0 0 3}$ \\
\hline 1,04 & $-27,56$ & 17,74 & $-9,30$ & $-23,57$ & $-1,50$ \\
\hline $\mathbf{2 0 0 4}$ & $\mathbf{2 0 0 5}$ & $\mathbf{2 0 0 6}$ & $\mathbf{2 0 0 7}$ & \multicolumn{1}{|c}{} \\
\cline { 1 - 3 } 19,20 & 12,05 & 29,04 & 29,26 & \multicolumn{1}{|c}{} \\
\cline { 1 - 2 } & & & &
\end{tabular}

Fuente: Ministerio de Desarrollo, Industria y Comercio Exterior de Brasil.

Cuanto a la participación de Argentina en el comercio exterior brasileño, fue ascendente hasta 1998, cuando se registró el nivel más alto en los últimos 22 años (ver Cuadros 3 y 4). Después, las exportaciones llegaron en el 2002 al índice más bajo en 12 años. A partir de entonces, se han recuperado y aproximado de los $9 \%$ en el 2007. Para las importaciones, la trayectoria ha sido descendente desde 2002, pero con el nivel siempre por arriba de los $8 \%$.

Cuadro 3 - Exportaciones brasileñas a Argentina (\% del total)

\begin{tabular}{|c|c|c|c|c|c|}
\hline 1986 & 1987 & 1988 & 1989 & 1990 & 1991 \\
\hline 3,04 & 3,17 & 2,90 & 2,10 & 2,05 & 4,67 \\
\hline 1992 & 1993 & 1994 & 1995 & 1996 & 1997 \\
\hline 8,49 & 9,49 & 9,50 & 8,69 & 10,83 & 12,78 \\
\hline 1998 & 1999 & 2000 & 2001 & 2002 & 2003 \\
\hline 13,20 & 11,17 & 11,32 & 8,60 & 3,88 & 6,24 \\
\hline 2004 & 2005 & 2006 & 2007 & & \\
\hline 7,64 & 8,38 & 8,52 & 8,97 & & \\
\hline
\end{tabular}

Fuente: Ministerio de Desarrollo, Industria y Comercio Exterior de Brasil. 
Cuadro 4 - Importaciones brasileñas de Argentina (\% del total)

\begin{tabular}{|l|l|l|l|l|l|}
\hline $\mathbf{1 9 8 6}$ & $\mathbf{1 9 8 7}$ & $\mathbf{1 9 8 8}$ & $\mathbf{1 9 8 9}$ & $\mathbf{1 9 9 0}$ & $\mathbf{1 9 9 1}$ \\
\hline 5,25 & 3,82 & 4,84 & 6,78 & 6,77 & 7,65 \\
\hline $\mathbf{1 9 9 2}$ & $\mathbf{1 9 9 3}$ & $\mathbf{1 9 9 4}$ & $\mathbf{1 9 9 5}$ & $\mathbf{1 9 9 6}$ & $\mathbf{1 9 9 7}$ \\
\hline 8,42 & 10,76 & 11,07 & 11,19 & 12,76 & 13,29 \\
\hline $\mathbf{1 9 9 8}$ & $\mathbf{1 9 9 9}$ & $\mathbf{2 0 0 0}$ & $\mathbf{2 0 0 1}$ & $\mathbf{2 0 0 2}$ & $\mathbf{2 0 0 3}$ \\
\hline 13,89 & 11,79 & 12,25 & 11,16 & 10,04 & 9,67 \\
\hline $\mathbf{2 0 0 4}$ & $\mathbf{2 0 0 5}$ & $\mathbf{2 0 0 6}$ & $\mathbf{2 0 0 7}$ & & \\
\cline { 1 - 2 } 8,86 & 8,48 & 8,82 & 8,63 & &
\end{tabular}

Fuente: Ministerio de Desarrollo, Industria y Comercio Exterior de Brasil.

Entre Brasil y Venezuela, ha ocurrido aumento en las exportaciones en 14 de los últimos 22 años e en las importaciones en 13, pero el aspecto más significativo es el crecimiento del comercio entre los dos países desde que Lula llegó al poder (ver Cuadros 5 y 6). En las exportaciones, fueron cuatro aumentos seguidos por arriba de los $30 \%$, incluido un de $141,79 \%$ en el 2004 , lo que significó un salto de US $\$ 799$ millones para US $\$ 4,72$ billones de 2002 a 2007. En las importaciones, hay dos elevaciones consecutivas por arriba de los $28 \%$, con destaque para una de $131,08 \%$ en el 2006 , después de una serie de cuatro caídas. La secuencia positiva se interrumpió en el 2007. Con Lula, el volumen del comercio entre los dos países pasó de US\$1,43 billón en el 2002 para US\$5,07 billones en el 2007.

Cuadro 5 - Exportaciones brasileñas a Venezuela (variación en \%)

\begin{tabular}{|c|c|c|c|c|c|}
\hline $\mathbf{1 9 8 6}$ & $\mathbf{1 9 8 7}$ & $\mathbf{1 9 8 8}$ & $\mathbf{1 9 8 9}$ & $\mathbf{1 9 9 0}$ & $\mathbf{1 9 9 1}$ \\
\hline 18,04 & 7,37 & 34,56 & $-47,25$ & 0,76 & 60,22 \\
\hline $\mathbf{1 9 9 2}$ & $\mathbf{1 9 9 3}$ & $\mathbf{1 9 9 4}$ & $\mathbf{1 9 9 5}$ & $\mathbf{1 9 9 6}$ & $\mathbf{1 9 9 7}$ \\
\hline 3,16 & $-11,15$ & $-28,33$ & 70,75 & $-5,51$ & 68,93 \\
\hline $\mathbf{1 9 9 8}$ & $\mathbf{1 9 9 9}$ & $\mathbf{2 0 0 0}$ & $\mathbf{2 0 0 1}$ & $\mathbf{2 0 0 2}$ & $\mathbf{2 0 0 3}$ \\
\hline$-7,99$ & $-24,00$ & 40,27 & 45,47 & $-27,05$ & $-23,87$ \\
\hline $\mathbf{2 0 0 4}$ & $\mathbf{2 0 0 5}$ & $\mathbf{2 0 0 6}$ & $\mathbf{2 0 0 7}$ & \multicolumn{1}{|l}{} \\
\cline { 1 - 3 } 141,65 & 51,29 & 60,34 & 32,49 & \multicolumn{1}{|l}{} &
\end{tabular}

Fuente: Ministerio de Desarrollo, Industria y Comercio Exterior de Brasil.

Cuadro 6 - Importaciones brasileñas de Venezuela (variación en \%)

\begin{tabular}{|c|c|c|c|c|c|}
\hline $\mathbf{1 9 8 6}$ & $\mathbf{1 9 8 7}$ & $\mathbf{1 9 8 8}$ & $\mathbf{1 9 8 9}$ & $\mathbf{1 9 9 0}$ & $\mathbf{1 9 9 1}$ \\
\hline$-62,92$ & 64,32 & $-11,37$ & 58,68 & 63,72 & 36,72 \\
\hline $\mathbf{1 9 9 2}$ & $\mathbf{1 9 9 3}$ & $\mathbf{1 9 9 4}$ & $\mathbf{1 9 9 5}$ & $\mathbf{1 9 9 6}$ & $\mathbf{1 9 9 7}$ \\
\hline$-22,45$ & 2,61 & 40,12 & 49,37 & 18,58 & 3,05 \\
\hline $\mathbf{1 9 9 8}$ & $\mathbf{1 9 9 9}$ & $\mathbf{2 0 0 0}$ & $\mathbf{2 0 0 1}$ & $\mathbf{2 0 0 2}$ & $\mathbf{2 0 0 3}$ \\
\hline$-24,90$ & 28,96 & 36,26 & $-43,74$ & $-15,25$ & $-56,54$ \\
\hline $\mathbf{2 0 0 4}$ & $\mathbf{2 0 0 5}$ & $\mathbf{2 0 0 6}$ & $\mathbf{2 0 0 7}$ & & \\
\hline$-27,65$ & 28,39 & 131,43 & $-41,52$ & & \\
\cline { 1 - 2 } & & &
\end{tabular}

Fuente: Ministerio de Desarrollo, Industria y Comercio Exterior de Brasil.

REVISTA DEBATES, Porto Alegre, v. 2, n. 2, p. 299-324, jul.-dez. 2008.310 
Cuanto a la participación en el comercio exterior brasileño, el crecimiento entre 2004 y 2007 ha levado las exportaciones para Venezuela a superar la marca de $2 \%$ del total por primera vez en 22 años, aproximándose de los 3\% en el 2007 (ver Cuadro 7). En las importaciones, los cinco años con los niveles más bajos del período analizado son con Lula en la Presidencia (ver Cuadro 8). Esto se reflecte en un saldo comercial muy favorable a Brasil desde que Lula asumió. EI superávit con Venezuela acumulado hasta 2007 es de US\$10,92 billones.

Cuadro 7 - Exportaciones brasileñas a Venezuela (\% del total)
\begin{tabular}{|c|c|c|c|c|c|}
\hline $\mathbf{1 9 8 6}$ & $\mathbf{1 9 8 7}$ & $\mathbf{1 9 8 8}$ & $\mathbf{1 9 8 9}$ & $\mathbf{1 9 9 0}$ & $\mathbf{1 9 9 1}$ \\
\hline 1,56 & 1,43 & 1,49 & 0,77 & 0,85 & 1,36 \\
\hline $\mathbf{1 9 9 2}$ & $\mathbf{1 9 9 3}$ & $\mathbf{1 9 9 4}$ & $\mathbf{1 9 9 5}$ & $\mathbf{1 9 9 6}$ & $\mathbf{1 9 9 7}$ \\
\hline 1,24 & 1,02 & 0,65 & 1,03 & 0,95 & 1,45 \\
\hline $\mathbf{1 9 9 8}$ & $\mathbf{1 9 9 9}$ & $\mathbf{2 0 0 0}$ & $\mathbf{2 0 0 1}$ & $\mathbf{2 0 0 2}$ & $\mathbf{2 0 0 3}$ \\
\hline 1,38 & 1,12 & 1,37 & 1,88 & 1,32 & 0,83 \\
\hline $\mathbf{2 0 0 4}$ & $\mathbf{2 0 0 5}$ & $\mathbf{2 0 0 6}$ & $\mathbf{2 0 0 7}$ & \\
\hline 1,52 & 1,88 & 2,59 & 2,94 & \\
\hline
\end{tabular}
Fuente: Ministerio de Desarrollo, Industria y Comercio Exterior de Brasil.

Cuadro 8 - I mportaciones brasileñas de Venezuela (\% del total)

\begin{tabular}{|c|c|c|c|c|c|}
\hline $\mathbf{1 9 8 6}$ & $\mathbf{1 9 8 7}$ & $\mathbf{1 9 8 8}$ & $\mathbf{1 9 8 9}$ & $\mathbf{1 9 9 0}$ & $\mathbf{1 9 9 1}$ \\
\hline 0,68 & 1,04 & 0,95 & 1,21 & 1,75 & 2,35 \\
\hline $\mathbf{1 9 9 2}$ & $\mathbf{1 9 9 3}$ & $\mathbf{1 9 9 4}$ & $\mathbf{1 9 9 5}$ & $\mathbf{1 9 9 6}$ & $\mathbf{1 9 9 7}$ \\
\hline 1,87 & 1,56 & 1,67 & 1,65 & 1,83 & 1,68 \\
\hline $\mathbf{1 9 9 8}$ & $\mathbf{1 9 9 9}$ & $\mathbf{2 0 0 0}$ & $\mathbf{2 0 0 1}$ & $\mathbf{2 0 0 2}$ & $\mathbf{2 0 0 3}$ \\
\hline 1,31 & 1,98 & 2,38 & 1,34 & 1,34 & 0,57 \\
\hline $\mathbf{2 0 0 4}$ & $\mathbf{2 0 0 5}$ & $\mathbf{2 0 0 6}$ & $\mathbf{2 0 0 7}$ & \multicolumn{2}{|l}{} \\
\cline { 1 - 4 } 0,32 & 0,35 & 0,65 & 0,29 & \multicolumn{2}{|r}{} \\
\cline { 1 - 3 } & & & &
\end{tabular}

Fuente: Ministerio de Desarrollo, Industria y Comercio Exterior de Brasil.

Otro indicador de la convergencia de posiciones en política exterior por parte de las tres potencias sudamericanas es las votaciones en la Asamblea General de las Naciones Unidas. De 2002, el año en que la página web del organismo empieza a traer el registro del voto por país, a 2007, el índice de coincidencia en las opciones de Argentina y Brasil ha estado siempre por arriba de los $80 \%$, con destaque para los dos últimos años (ver Cuadro 9). 
Cuadro 9 - Votos coincidentes entre Argentina y Brasil en la ONU (\%)

\begin{tabular}{|l|l|l|l|l|l|}
\hline $\mathbf{2 0 0 2}$ & $\mathbf{2 0 0 3}$ & $\mathbf{2 0 0 4}$ & $\mathbf{2 0 0 5}$ & $\mathbf{2 0 0 6}$ & $\mathbf{2 0 0 7}$ \\
\hline 88,00 & 82,50 & 80,20 & 84,60 & 88,10 & 88,80 \\
\hline \multicolumn{6}{c|}{ Fuente: ONU. }
\end{tabular}

Entre Brasil y Venezuela, los índices de coincidencia también son muy altos $y$, en promedio, incluso superan los de argentinos y brasileños (ver Cuadro 10). De 2002 a 2007, han estado siempre por arriba de los $82,50 \%$, con el ápice en los $91,75 \%$ de 2003 , cuando empezó el primer gobierno Lula.

Cuadro 10 - Votos coincidentes entre Brasil y Venezuela en la ONU (\%)

\begin{tabular}{|l|l|l|l|l|l|}
\hline $\mathbf{2 0 0 2}$ & $\mathbf{2 0 0 3}$ & $\mathbf{2 0 0 4}$ & $\mathbf{2 0 0 5}$ & $\mathbf{2 0 0 6}$ & $\mathbf{2 0 0 7}$ \\
\hline 86,10 & 91,75 & 83,50 & 82,70 & 84,40 & 87,75 \\
\hline
\end{tabular}

Fuente: ONU.

Cuando se trata de la coincidencia de votos entre Argentina y Venezuela, los índices caen un poco, pero han sido siempre iguales o mayores que 75\% (ver Cuadro 11). La aproximación política reciente entre Kirchner y Chávez se reflecte en el resultado de los dos últimos años, los más altos del período analizado.

Cuadro 11 - Votos coincidentes entre Argentina y Venezuela en la ONU (\%)

\begin{tabular}{|c|c|c|c|c|c|}
\hline $\mathbf{2 0 0 2}$ & $\mathbf{2 0 0 3}$ & $\mathbf{2 0 0 4}$ & $\mathbf{2 0 0 5}$ & $\mathbf{2 0 0 6}$ & $\mathbf{2 0 0 7}$ \\
\hline 77,80 & 79,40 & 75,80 & 75,00 & 82,60 & 83,70 \\
\hline \multicolumn{6}{c|}{ Fuente: ONU }
\end{tabular}

Una última evidencia es los préstamos de fondos públicos brasileños en Argentina y Venezuela. Es necesario a un proyecto integracionista entre países dispares que los Estados de más capacidad económica asuman el papel de financiar el proceso y amenizar las asimetrías, transfiriendo recursos a los menos desarrollados. Ha ocurrido históricamente presión interna e externa para Brasil ejercer esta función. Aunque tímidamente, el gobierno Lula ha actuado en este sentido, condicionando los préstamos al uso de bienes o servicios brasileños en los proyectos (ver Cuadro 12). Para tanto, incluso, fue creado el Departamento de Integración de la América del Sur en el BNDES (Banco Nacional de Desarrollo Económico y Social). 


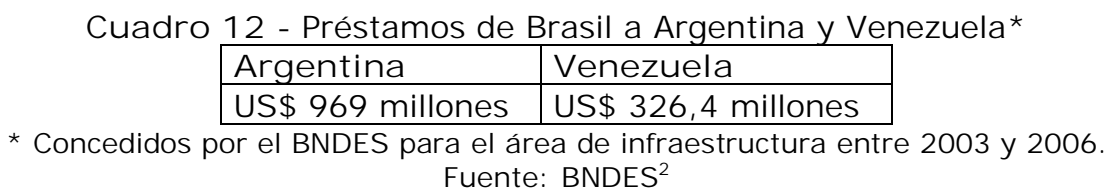

\section{La Evolución de las Relaciones entre Argentina y Brasil}

Como Argentina y Brasil son las dos potencias de América del Sur, hay que se dar atención a ellos en cualquier tema relacionado al subcontinente. Además, parte fundamental de las políticas externas de ambos para la integración regional es la relación bilateral. Sin el entendimiento entre argentinos y brasileños, las bases de la integración sudamericana se quedan comprometidas. Así, la evolución de las relaciones entre los dos países tiene que ser otra variable independiente, aunque el papel específico de Argentina en la creación de UNASUR haya sido menos importante que los de Brasil y Venezuela.

La cooperación entre Argentina y Brasil ha sido facilitada a partir del acuerdo sobre la Bacía del Plata. Desde entonces, han ocurrido momentos de más o menos proximidad. En el período de Menem, el MERCOSUR surgió y evolucionó, pero con énfasis comercial y como parte del programa de reformas neoliberales. Al mismo tiempo, se retomó la idea de hacer de Argentina el aliado preferencial de Estados Unidos en América del Sur. Fue a partir del gobierno Duhalde que las relaciones con Brasil se han tornado prioridad. Con las llegadas de Kirchner y Lula al poder en el 2003, esto se ha reforzado.

Además de lo que ha sido expuesto en el último apartado, hay otros indicadores para sustentar estas evaluaciones. En los 20 años de régimen militar en Brasil, de 1964 a 1984, el promedio de acuerdos con Argentina, que también estuvo bajo dictadura de 1966 a 1973 y de 1976 a 1983, fue de 2,33. En el intervalo entre 1985, cuando los dos ya están redemocratizados, y 2007, el promedio salta para 7,35 (ver Cuadros 13 y 14$)$.

\footnotetext{
${ }^{2}$ Los datos provienen de textos noticiosos en la página web de BNDES y de artículos en la prensa cuya origen de las informaciones también es el banco.
} 
Cuadro 13 - Acuerdos entre Brasil y Argentina durante el régimen militar brasileño*

\begin{tabular}{|c|c|c|c|c|c|c|}
\hline 1964 & 1965 & 1966 & 1967 & 1968 & 1969 & 1970 \\
\hline 1 & 1 & 0 & 0 & 2 & 1 & 1 \\
\hline 1971 & 1972 & 1973 & 1974 & 1975 & 1976 & 1977 \\
\hline 2 & 4 & 0 & 1 & 1 & 0 & 1 \\
\hline 1978 & 1979 & 1980 & 1981 & 1982 & 1983 & 1984 \\
\hline 0 & 1 & 23 & 1 & 2 & 3 & 4 \\
\hline \multicolumn{7}{c|}{ * Incluye acuerdos en el ámbito de la Bacía del Plata. I tamaraty. }
\end{tabular}

Cuadro 14 - Acuerdos entre Brasil y Argentina desde la redemocratización en los dos*

\begin{tabular}{|c|c|c|c|c|c|c|c|}
\hline 1985 & 1986 & 1987 & 1988 & 1989 & 1990 & 1991 & 1992 \\
\hline 5 & 10 & 4 & 6 & 7 & 11 & 9 & 1 \\
\hline 1993 & 1994 & 1995 & 1996 & 1997 & 1998 & 1999 & 2000 \\
\hline 9 & 6 & 4 & 11 & 17 & 5 & 5 & 8 \\
\hline 2001 & 2002 & 2003 & 2004 & 2005 & 2006 & 2007 & \\
\hline 5 & 5 & 10 & 2 & 23 & 3 & 3 & \\
\hline
\end{tabular}

Al analizarse cada gobierno en los dos países desde la redemocratización, también se ve una evolución del promedio de tratados, que va, respectivamente, de 5,27 y 6,40 con Alfonsín y Sarney hasta 8,73 y 9,50 con Kirchner y Lula (ver Cuadros 15 y 16). Las gestiones con mayor promedio de acuerdos en cada país fueron la segunda de Menem y la primera de Lula. El primer año del segundo período menemista marcó el inicio de la fase de zona aduanera del MERCOSUR. A pesar de esto, la mayoría de los tratados en su segundo mandato no son en el ámbito del bloque. Es curioso que la administración que más celebró acuerdos con Brasil sea de Menem, cuyo canciller Guido Di Tella abogó por relaciones carnales con Estados Unidos, y que el MERCOSUR no sea la explicación. Después del segundo gobierno Menem y del primer de Fernando Henrique, hubo una caída en el número de tratados hasta las ascensiones de Kirchner y Lula, que tienen, respectivamente, el segundo e el primer mayor promedio de cada país. Con los dos, 2005 fue el año con más acuerdos del período democrático. 
Cuadro 15 - Promedio de acuerdos con Argentina por gobierno democrático en Brasil

\begin{tabular}{|c|c|c|}
\hline Sarney (1985-1989) & Collor (1990-1992) & I tamar* (1992-1994) \\
\hline 6,40 & 7,00 & 7,50 \\
\hline FHC 1 (1995-1998) & FHC 2 (1999-2002) & Lula 1 $(2003-2006)$ \\
\hline 9,25 & 5,75 & 9,50 \\
\hline \multirow{3}{*}{ *El año de 1992 es computado para Collor pues el gobierno Itamar empezó el 2/10. } \\
Fuente: Itamaraty.
\end{tabular}

Cuadro 16 - Promedio de acuerdos con Brasil por gobierno democrático en Argentina

\begin{tabular}{|c|c|c|}
\hline Alfonsín* (1983-1989) & Menem 1 (1989-1995) & Menem 2 (1995-1999) \\
\hline 5,27 & 7,17 & 9,33 \\
\hline De la Rúa** (1999-2001) & Duhalde (2002-2003) & Kirchner (2003-2007) \\
\hline 6,50 & 4,22 & 8,73 \\
\hline
\end{tabular}

* El año de 1983 no entra en el cálculo pues el gobierno empezó el 10/12.

** El año de 1999 es computado para Menem 2 pues el gobierno De la Rúa empezó el $10 / 12$

Fuente: Itamaraty.

\section{EI Surgimiento de un Líder Pro Integración en Venezuela}

Como Venezuela históricamente ha priorizado las relaciones con el norte de América del Sur, el Caribe y los Estados Unidos, el proyecto integracionista sudamericano carecía de un gobierno venezolano que le fuera más favorable. La posición chavista pro integración es evidente no por la retórica contra Estados Unidos. Lo principal ha sido hecho en términos prácticos. En un país que es el séptimo mayor productor petrolífero del mundo e exporta alrededor de $70 \%$ de su producción, los récords en los precios del petróleo han permitido a Chávez acumular reservas también para su política exterior.

Así, Venezuela se ha convertido en financiadora del proceso de integración, junto con Brasil. Chávez practica una diplomacia del petróleo, ofreciendo el producto a mejores precios, transferencia de tecnología e iniciativas conjuntas en América del Sur y Latina. Entre los países sudamericanos, hay negociaciones para conceder beneficios y acciones bilaterales en curso con al menos Argentina, Brasil, Bolivia, Ecuador, Paraguay y Uruguay. En ayudas financieras, algunos números ilustran lo que ha ocurrido entre Argentina y Venezuela. En el gobierno Kirchner, hubo la compra de US\$ 5,2 billones de bonos de la deuda del país. Hasta mediados de 2007, ya había acuerdos para prestar US\$ 535 millones a proyectos en Argentina. Además, los dos países se 
complementan económicamente, pues los argentinos necesitan de energía, y los venezolanos, de tecnologías agrícola e industrial.

En un bloque con fuertes disparidades entre sus miembros, es necesario que los Estados de más capacidad económica asuman el papel de financiar el proceso e amenizar las asimetrías, fomentando el desarrollo regional y transfiriendo recursos a los menos desarrollados. En la UE (Unión Europea), esta función cabe, sobre todo, a Alemania. En América del Sur, hay presión para que Brasil desempeñe el papel, pero el país no lo ha asumido integralmente hasta ahora. La evaluación de que Brasil precisa se comprometer con mecanismos obligatorios de transferencia de recursos, como los fondos estructurales de la UE, no tiene la misma fuerza en su diplomacia que la posición favorable a la integración sudamericana, mismo en el gobierno Lula, porque hay la percepción de que el país todavía no reúne las condiciones para tal. Así, la actuación de la Venezuela de Chávez en la financiación del proceso es útil al Brasil de Lula.

Hay quien estima que Chávez, por los problemas que su busca de protagonismo crea, es más prejudicial que útil a la integración en América del Sur y que su proyecto integracionista, que es para América Latina, rivaliza con el sudamericano. Las dos evaluaciones parecen equivocadas. En primer lugar, es evidente que la retórica chavista y la tentativa de influenciar la política interna de otros países sudamericanos generan problemas, pero mientras él tenga el dinero del petróleo para repartir, esto tiende a quedar en segundo plano. El antagonismo de proyectos tampoco se sostiene, porque Chávez llevó Venezuela al MERCOSUR y es un entusiasta de la integración sudamericana, con iniciativas como el Banco del Sur y la PETROSUR negociadas justamente por Argentina, Brasil y Venezuela, entre otros. Es cierto que también sacó su país de la CAN (Comunidad Andina de Naciones), pero con una justificativa que es más bien un apoyo a las iniciativas regionales que el contrario, diciendo que el bloque no tenía más sentido después de los tratados de libre comercio de Colombia y Perú con Estados Unidos.

El organismo que consubstancia el proyecto integracionista de Chávez para América Latina, la ALBA (Alternativa Bolivariana para los 
Pueblos de Nuestra América), puede y ha seguido paralelamente a UNASUR, pues cumplen funciones diferentes e al mismo tiempo complementares. El primer se concentra en el ámbito de la retórica contra los Estados Unidos y la globalización neoliberal, a pesar de que también ha evolucionado en términos prácticos, y sirve como espacio privilegiado para el discurso chavista y el ejercicio de su política exterior. Ya la UNASUR busca la institucionalización gradual de un bloque regional y requiere la formación de consensos. Así, la ALBA es útil para amenizar la necesidad de protagonismo de Chávez, contribuyendo para que él se contente en ser una pieza importante en UNASUR.

Es evidente que la actuación chavista continuará a generar problemas, pero esto tiende a ser circunstancial mientras el dinero del petróleo permita a él seguir como financiador en la región y las tres potencias continúen a preocuparse en superar divergencias en nombre del objetivo mayor de la integración.

Las iniciativas que Chávez defiende para la integración sudamericana también sirven para saltar etapas. Como la acción diplomática suele ser formal y lenta, hasta por la naturaleza de la actividad, el abordaje directo de la política exterior chavista tiene esta utilidad. Al mismo tiempo, la connotación política que él da a las iniciativas crea dificultades para la adhesión de todos los países.

\section{La Evolución de UNASUR}

Antes de la reunión en que se creó la UNASUR, ocurrieron dos cumbres sudamericanas de presidentes, en el 2000 en Brasilia e en el 2002 en Guayaquil, Ecuador, durante el segundo gobierno de Fernando Henrique. En ambas, ya se trató de la integración sudamericana. La declaración final de la I Cumbre estableció 2002 como límite para la creación de una zona de libre comercio entre MERCOSUR y CAN, lo que tardó hasta 2005, y definió la “formação de um espaço econômico- 
comercial ampliado na América do Sul ${ }^{3 \prime}$ como un objetivo. El texto, sin embargo, dio apoyo al avance de la ALCA, desde que viniese a asegurar acesso efetivo a las exportaciones sudamericanas. Por otro lado, anunció que los países de la región pasarían a negociar el tema en conjunto y citó las cuestiones del tráfico de drogas y del terrorismo, otros puntos caros a Estados Unidos.

El documento final de la II Cumbre trae un cambio de énfasis que marcaría la visión de la integración sudamericana por el gobierno Lula. En lugar de un área económico-comercial, se refiere a un espacio integrado por el fortalecimiento "das conexões físicas e da harmonização dos aspectos institucionais, normativos e regulatórios ${ }^{4 "}$. La ALCA todavía aparece, pero sin apoyo. Las menciones a ella desaparecerían a partir de ahí, así como su negociación.

La superación del ámbito comercial fue profundada en la Declaración de Cusco, que creó la UNASUR. El texto final de la III Cumbre establece como objetivos del organismo la coordinación política, la extensión de la zona de libre comercio a toda América del Sur y la integración económica, social, institucional, física, energética y de comunicaciones. La declaración es contraria a una estructura institucional propia para el bloque, alegando que utilizar la ya existente en otros organismos evitaría superposición y nuevos gastos. Al mismo tiempo, establece las cumbres de jefes de Estado como instancia máxima de UNASUR y las de cancilleres para las decisiones ejecutivas y crea una Presidencia Pro Tempore del bloque a cargo de un Estado miembro por turno.

En la I Cumbre de Jefes de Estado de la UNASUR, en el 2005, fueron definidos el Programa de Acción y la Agenda Prioritaria, que fija la periodicidad de un año para las cumbres y define que la Presidencia

\footnotetext{
${ }^{3}$ Comunicado de Brasília, 01/09/2000. Disponible en: <http://www.radiobras.gov.br/integras/00/integra_0109_3.htm>. Acceso en: 03 mar. 2007.

${ }^{4}$ Consenso de Guaiaquil sobre Integração, Segurança e Infra-Estrutura para o Desenvolvimento, 27/07/2002. Disponible en: <http://www2.mre.gov.br/daa/consenso guaiaquil.htm>. Acceso en: 03 mar. 2007.
} 
Pro Tempore será ejercida en el período entre una y otra reunión presidencial por el país sede de la última.

La declaración final de la II Cumbre, en el 2006, se refiere a una "ciudadanía sudamericana", con "derechos civiles, políticos, laborales y sociales a los nacionales de un Estado miembro en cualquiera de los otros". El primer ato jurídico internacional emanado de UNASUR es justamente un acuerdo de 2006 para dispensa de visado y utilización de documento simple de identificación por los nacionales de los Estados miembros en los países del bloque. La declaración final también crea la Comisión de Altos Funcionarios, para aplicar las decisiones ejecutivas, profundizar los vínculos entre CAN y MERCOSUR y preparar el Tratado Constitutivo; facilita a la comisión un cuerpo técnico y una sede provisoria en Río de Janeiro; y sugiere el estudio de la creación de un Parlamento sudamericano.

Ya el texto de la Comisión de Reflexión presentado en la II Cumbre trae un anexo del Grupo de Trabajo sobre Integración Financiera. Su principal sugerencia es la creación gradual del Banco del Sur, defendido por el gobierno Chávez para financiar proyectos de infraestructura y programas sociales y actuar en crisis especulativa o de solvencia, incluso en el ámbito latinoamericano. Sin embargo, había discordancia con Brasil. Argentina, en el anexo, afirma estar de acuerdo con Brasil, pero adhirió después a la propuesta chavista. A finales de 2007, se llegó a un acuerdo para la formación del Banco del Sur, con siete (Argentina, Bolivia, Brasil, Ecuador, Paraguay, Uruguay y Venezuela) de los 12 países de América del Sur. Brasil logró limitar la actuación de la nueva institución al financiamiento de proyectos de infraestructura y programas sociales y al ámbito sudamericano.

La primera cumbre sudamericana de presidentes, en el 2000, ya había creado la IIRSA (Iniciativa para la Integración de la Infraestructura Regional Suramericana), responsable por proyectos conjuntos de infraestructura. Una obra en ejecución es la Carretera

5 Declaración de Cochabamba, 09/12/2006. Disponible en: <http://www.comunidadandina.org/documentos/dec_int/ declaracion_cochabamba.htm> Acceso en: 03 mar. 2007. 
Interoceánica, para unir Perú y Brasil pasando por Bolivia. A pesar de merecer atención en las reuniones de UNASUR, la IIRSA, así como el Banco del Sur, no aparece en el Tratado Constitutivo.

En la I Cumbre Energética Sudamericana, en el 2007 en Venezuela, se decidió crear un consejo del área; cambiar el nombre de la CASA para UNASUR; instituir una Secretaría General, en Quito, Ecuador; agregar la Comisión de Altos Funcionarios a esta Secretaría; y determinar la conclusión del Tratado Constitutivo hasta la III Cumbre de Jefes de Estado, marcada para 2007 en Cartagena de Indias, Colombia. Este encuentro acabó convertido en reunión extraordinaria y realizado en el 2008 en Brasilia a causa del retraso en la preparación del tratado y de una acción militar colombiana en territorio ecuatoriano.

El tercer encuentro marcó la firma del Tratado Constitutivo, que confiere carácter jurídico internacional a UNASUR. El documento reafirma principios, como el respecto a la soberanía y a la inviolabilidad territorial, en tiempos de la tentativa colombiana de importar la táctica norteamericana de acción preventiva, y metas, como la construcción de un espacio "integrado no âmbito político, econômico, social, cultural, ambiental, energético e de infra-estrutura6". Entre los objetivos específicos, están la integración financiera, que deja abierta, sin la mencionar, la posibilidad de unión monetaria; la adopción de mecanismos para amenizar las asimetrías; la cooperación entre autoridades judiciales; y el intercambio en el tema de la defensa, lo que ya ha generado la discusión de la creación de un consejo.

Además, el tratado establece la estructura institucional del bloque, formada por el Consejo de Jefas e Jefes de Estado y de Gobierno, el Consejo de Ministras y Ministros de las Relaciones Exteriores, el Consejo de Delegadas y Delegados y la Secretaría General, el único de carácter supranacional, y fija las funciones y la periodicidad de reuniones de cada órgano. El primer consejo, la instancia máxima, se reúne anualmente y tiene la pauta definida por el

\footnotetext{
${ }^{6}$ Tratado Constitutivo da União de Nações Sul-Americanas, 23/05/2008. Disponible en: <http://www.mre.gov.br/ portugues/imprensa/nota_detalhe3.asp?ID_RELEASE=5466> Acceso en: 24 may. 2008.
} 
órgano ministerial, que, por su turno, depende del Consejo de Delegados para la definición de su propia pauta. La Secretaría General también puede proponer iniciativas y actuar en las reuniones de los demás órganos. Las normas se adoptan por consenso, pero la deliberación puede ocurrir con la presencia de tres cuartos de los Estados miembros, y son de cumplimiento obligatorio, pero su incorporación a los ordenamientos nacionales, la condición de efectividad, no es automática. Hay la posibilidad de que tres o más Estados miembros desarrollen, sin la participación de los demás, una iniciativa aprobada por la instancia máxima. Los ausentes pueden adherir después. Para la solución de controversias sobre el tratado, no se funda un órgano específico. Finalmente, el documento determina la creación de un Parlamento Sudamericano, en Cochabamba, por medio de un protocolo adicional.

La convergencia institucional entre CAN y MERCOSUR es un pilar de lo que se ha planteado en las cumbres de UNASUR para el desarrollo del organismo. Esto, sin embargo, es problemático, porque el bloque andino tiene rasgos más supranacionales que el del Cono Sur (OLIVEIRA e ONUKI, 2006).

\section{Conclusiones}

Lo expuesto hasta aquí sustenta la hipótesis de que la convergencia de posiciones integracionistas en las tres potencias regionales, impulsada por un canciller y dos presidentes, posibilitó la creación de UNASUR. Algunos datos que atestan esto son: 1) el promedio de acuerdos entre Argentina y Brasil saltó de 2,33 durante el régimen militar brasileño para 7,35 entre 1985 y 2007; 2) la primera administración de Lula y la de Kirchner tienen el año con más tratados del período democrático y, respectivamente, el primer e el segundo mayor promedio; 3) el gobierno Chávez prestó u invirtió al menos US\$ 5,735 billones en Argentina de 2005 a 2007; 4) la gestión Lula prestó al menos US\$ 1,295 billón a argentinos y venezolanos entre 2003 y 2006; 5) las exportaciones brasileñas a Argentina han aumentado en 15 de los últimos 22 años e en todos los cinco con Lula, mientras las argentinas a 
Brasil, en 17 de los 22 e en cuatro de los cinco con Lula; 6) el volumen del comercio bilateral desde que Lula llegó a la Presidencia pasó de US\$ 7,09 billones en el 2002 para US\$ 24,83 billones en el 2007; 7) la participación argentina en el comercio exterior brasileño fue ascendente hasta 1998 y se ha recuperado en las exportaciones desde 2003; 8) en las transacciones con Venezuela, ha ocurrido aumento de las ventas brasileñas en 14 de los últimos 22 años y de las importaciones en 13; 9) con Lula, el volumen comercial entre los dos países pasó de US\$1,43 billón en el 2002 para US\$5,07 billones en el 2007, y las ventas a Venezuela han superado $2 \%$ del total exportado por primera vez en 22 años; 10) la coincidencia de votos entre Argentina y Brasil en la Asamblea General de la ONU ha sido siempre superior a $80 \%$ de 2002 a 2007, con promedios de casi $84 \%$ para Kirchner e el primer gobierno Lula; 11) con Venezuela, la coincidencia se ha mantenido por arriba de los $82,50 \%$, con promedios en torno de $86 \%$ para Lula y Chávez; y 12) entre Argentina y Venezuela, el índice ha sido siempre igual o mayor que $75 \%$, con promedios entre $77 \%$ y poco más de $78 \%$ para Kirchner y Chávez.

Estos datos están alineados con la evaluación de que el escenario se ha tornado más favorable a la integración sudamericana a partir de 2003, cuando Kirchner y Lula se juntaron a Chávez como presidentes de sus respectivos países. A las actuaciones sobre todo de los dos últimos, como también muestran los datos, a favor de la unión de fuerzas en América del Sur y de la creación de un organismo regional, hay que añadir el papel del canciller brasileño, Celso Amorim, el primero a presentar una propuesta oficial en esta dirección. Al mismo tiempo que se ha fortalecido la posición integracionista en las tres potencias, se han enflaquecido los defensores de relaciones especiales con Estados Unidos. Una evidencia del segundo punto es el fracaso del proyecto norteamericano de la ALCA, sobre todo por la acción de los países alineados en torno del MERCOSUR.

El factor más importante a impulsar la integración sudamericana ha sido la acción gubernamental. A causa del estadio en que el proceso se encuentra y del enfoque en el período que antecede la creación de 
UNASUR, no sería adecuado seguir una línea neofuncionalista y buscar por los aspectos que impulsan la integración desde instituciones supranacionales y grupos de la sociedad civil, organizados o no. Hasta en el MERCOSUR, más desarrollado institucionalmente, todavía no se puede identificar una fuerza interna significativa a punto de influenciar sus rumbos. Esto se pasa, entre otros motivos, porque gran parte de su burocracia todavía tiene dupla función, en el aparato administrativo del Estado a que se vincula e en el cuerpo técnico o ejecutivo del bloque.

Así, la línea seguida en este estudio parece ser la más apropiada, con la elección de variables independientes relacionadas a la acción gubernamental y la conclusión de que la convergencia de posiciones integracionistas en las tres potencias regionales, sobre todo en Brasil y Venezuela, ha posibilitado la conformación de UNASUR.

João Carlos Amoroso Botelho é doutorando em Ciência Política pela Universidade de Salamanca/Espanha e professor de Relações Internacionais na Pontifícia Universidade Católica de São Paulo.

E-mail: joaocarlosbotelho@hotmail.com

\section{Referencias:}

BRASIL. División de Actos Internacionales. In: MINISTERIO DE LAS RELACIONES EXTERIORES - Itamaraty. Disponible en: <http://www2.mre.gov.br/dai/home.htm>. Acceso en: 16 jun. 2008.

Estadísticas sobre el comercio exterior brasileño. In: MINISTERIO DE DESARROLLO, INDUSTRIA Y COMERCIO EXTERIOR. Disponible en: <http://www. desenvolvimento.gov.br/sitio/interna/interna. php?area =5 \&menu=576> y <http://aliceweb.desenvolvimento.gov.br/default.asp>. Acceso en: 17 jun. 2008.

CAPORASO, James. Regional integration theory: Understanding our past and anticipating our future. En: SANDHOLTZ, Wayne; STONE SWEET, Alec (Eds.). European integration and supranational governance. Oxford: Oxford University Press, 1998. p. 334-351. 
CERVO, Amado Luiz. Relações internacionais da América Latina: Velhos e novos paradigmas. São Paulo: Ática, 2007.

COMUNICADO DE BRASÍLIA. 01 sep. 2000. Disponible en: <http://www.radiobras.gov.br/integras/00/integra_0109_3. htm>. Acceso en: 03 mar. 2007.

CONSENSO de Guaiaquil sobre Integração, Segurança e Infra-Estrutura para o Desenvolvimento. 27 jul. 2002. Disponible en: <http://www2.mre.gov.br/daa/consenso guaiaquil.htm>. Acceso en: 03 mar. 2007.

CORRÊA, Luiz Felipe de Seixas. Um continente em desenvolvimento. In: CERVO, Amado Luiz. Relações internacionais da América Latina: Velhos e novos paradigmas. São Paulo: Ática, 2007. p. XI-XIII.

COSTA VAZ, Alcides. Cooperação, integração e processo negociador: A construção do Mercosul. Brasilia: IBRI, 2002.

DECLARACIÓN DE COCHABAMBA. 09 dic. 2006. Disponible en: <http: //www.comunidadandina.org/documentos/dec_int/declaracion_co chabamba.htm>. Acceso en: 03 mar. 2007.

HAAS, Ernst. The uniting of Europe: Political, social, and economic forces (1950-57). Stanford: Stanford University Press, 1958.

OLIVEIRA, Amâncio Jorge de; ONUKI, Janina. Entraves e possibilidades institucionais e políticos da integração regional sul-americana. Ponencia presentada al III Congreso Latinoamericano de Ciencia Política, 2006. Campinas. CD Rom - Democracia e Desigualdades.

ONU. Resoluciones de la Asamblea General de las Naciones Unidas. In: ORGANIZACIÓN DE LAS NACIONES UNIDAS. Disponible en: <http://www.un.org/Depts/dhl/spanish/resguids/gareslsp.htm>.

Acceso en: 06 oct. 2008.

SCHMITTER, Philippe. Examining the present euro-polity with the help of past theories. En: MARKS, Gary; SCHARPF, Fritz; SCHMITTER, Philippe; STREECK, Wolfgang. Governance in the European Union. Londres: Sage Publications, 1996. p. 1-14.

TRATADO CONSTITUTIVO DA UNIÃO DE NAÇÕES SUL-AMERICANAS. 23 may. 2008. Disponible en: <http://www.mre.gov.br/ portugues/imprensa/nota_detalhe3.asp?ID_RELEASE $=5466>$. Acceso en: 24 may. 2008. 